\title{
MiR-548c impairs migration and invasion of Cossmak endometrial and ovarian cancer cells via downregulation of Twist
}

\author{
Xiaochun Sun ${ }^{1}$, Manhua Cui ${ }^{2}$, Aichen Zhang ${ }^{1}$, Lingling Tong ${ }^{1}$, Kun Wang ${ }^{1}$, Kai Li ${ }^{1}$, Xue Wang ${ }^{1}$, Ziqian Sun ${ }^{1}$ \\ and Hongye Zhang ${ }^{1}$
}

\begin{abstract}
Background: MicroRNAs (miRNAs) are a class of small non-coding RNAs, which post-transcriptionally repress the expression of genes involved in cancer initiation and progression. Although some miRNAs that target many signaling pathways (also called universe miRNAs) are supposed to play a global role in diverse human tumors, their regulatory functions in gynecological cancers remain largely unknown. We investigated the biological role and underlying mechanism of miR-548c (one universe miRNA) in endometrial and ovarian cancer.

Methods: The effects of miR-548c overexpression on cell proliferation, migration and invasion were studied in endometrial and ovarian cancer cells. TWIST1 (Twist) was identified as a direct miR-548c target by western blot analysis and luciferase activity assay. The expression of miR-548c and Twist were examined by qRT-PCR in endometrial and ovarian cancer tissues.

Results: Here, we report that miR-548c is down-regulated in endometrial and ovarian cancer tissues when compared to normal tissues, and our meta-analysis reveal that decreased miR-548c expression correlates with poor prognosis in endometrial cancer patients. We show that in endometrial and ovarian cancer cells, ectopic expression of miR-548c significantly inhibits whereas knockdown of miR-548c dramatically induces cancer cell proliferation, migration and invasion. By using luciferase reporter assay, we demonstrate that Twist, a known oncogene in endometrial and ovarian cancers, is a direct target of miR-548c. Furthermore, the expression of Twist partially abrogates the tumor suppressive effects of miR-548c on cell migration and invasion.

Conclusion: These findings suggest that miR-548c directly downregulates Twist, and provide a novel mechanism for Twist upregulation in both endometrial and ovarian cancers. The use of miR-548c may hold therapeutic potential for the treatment of Twist-overexpressing tumors.
\end{abstract}

Keywords: microRNA, miR-548c, EMT, Endometrial cancer, Ovarian cancer

\section{Background}

Metastasis is the main cause of death among patients with endometrial cancer $(\mathrm{EC}$, the most frequently diagnosed gynecologic malignancy) cbl1, 2] and ovarian cancer (OC, the most lethal gynecological cancer) [3]. The initial steps of metastasis include the detachment and migration of cancer cells and subsequent invasion into surrounding tissues [4]. Epithelial-to-mesenchymal

\footnotetext{
* Correspondence: cuimanhua@outlook.com

${ }^{2}$ Department of Obstetrics and Gynecology, the Second Hospital of Jilin University, Changchun 130041, China

Full list of author information is available at the end of the article
}

transition (EMT) is considered a key step in metastasis of endometrial and ovarian cancer $[1,5,6]$, which enhances the migratory and invasive capacity of tumor cells to facilitate malignant progression. Therefore, understanding the molecular mechanisms that mediate migration, invasion and EMT process of EC and OC cells could identify novel molecular targets for future treatments of these cancers.

Twist1 (Twist) is a critical oncogene that is overexpressed and plays important roles in EMT induction in EC and OC $[1,7,8]$. High Twist expression positively correlated with deep myometrial invasion and poor 
outcome in EC [9]. Aberrant expression of Twist in OC correlated with advanced tumor stage and predicted a poor clinical outcome [10, 11]. MicroRNAs (miRNAs) are a class of small non-coding RNA molecules that regulate gene expression via interactions with the 3'-untranslated regions (UTRs) of mRNAs targets, causing translational suppression or mRNA decay. MiRNAs control metastatic progression [12, 13], and act as either tumor suppressors or oncogenes in EC and OC $[1,14]$. Although a number of miRNAs, such as miR-106b and miR-543, have been reported to suppress the expression of Twist and EC cell invasion $[15,16]$, miRNAs affecting Twist expression in EC and OC is still not completely understood. Recently, two distinct classes of microRNAs: universe miRNAs (for example, miR-34a) that regulate many signaling pathways, as well as intra-pathway miRNAs that target multiple genes within a single signaling pathway, have been identified [17]. Universe microRNAs are predicted to affect more targets/signaling pathways, and might play a global role in tumor cells [17]. Of interest, several universe miRNAs (including miR-548a/ $\mathrm{b} / \mathrm{c}$, etc.) belong to the same miR-548 family [17] and have been implicated in tumorigenesis $[18,19]$, however their regulatory functions in gynecological cancers remain largely unknown.

In this study, we investigated the biological functions and underlying mechanism of miR-548c in EC and $\mathrm{OC}$, and provide in vitro evidence that miR-548c inhibits migration and invasion in both $\mathrm{EC}$ and $\mathrm{OC}$ cells by directly targeting Twist. We further demonstrate that miR-548c expression inversely correlates with Twist expression in EC and $\mathrm{OC}$ tissues and the downregulation of miR-548c is associated with poor prognosis in EC patients.

\section{Methods}

\section{Cell culture and transient transfection}

The human EC cell lines (RL95-2 and HEC-1) and human OC cell lines (SKOV-3 and OVCAR3) were obtained from Chinese Academy of Sciences Committee on Type Culture Collection Cell Bank, Shanghai, China. These cells were cultured in DMEM/F12 medium (Invitrogen, Shanghai) or PRMI-1640 medium (Gibco, Carlsbad, CA, USA) supplemented with $10 \%$ fetal bovine serum (FBS, Invitrogen, Shanghai). MiR-548c mimic $(40 \mathrm{nM})$, antisense miR-548c inhibitor (40 nM), Twist siRNA ( $5 \mathrm{nM})$ and respective negative controls were purchased from Ambion (TX, USA), and were transfected using Lipofectamine 2000 (Invitrogen, CA, USA), according to the manufacturer's instructions. Transient transfection of Twist cDNA plasmids (OriGene, MD, USA) were performed with Lipofectamine Plus reagent (Invitrogen, CA, USA) according to the manufacturer's protocol.

\section{RNA extraction and real-time qRT-PCR}

Total RNA was extracted using TRIzol reagents (Invitrogen, CA, USA). The miRNA qRT-PCR was performed using the NCode miRNA qRT-PCR analysis (Invitrogen, CA, USA). Forward primers for miRNA detection are the exact sequence of mature miR-548c-3p. For mRNA analysis, RT reaction was carried out using $100 \mathrm{ng}$ total RNA with the PrimeScript RT reagent kit (Takara, Japan). Real-time qRT-PCR was performed using the Takara SYBR Premix Ex Taq II (Takara, Japan). Primers used for the amplification of E-cadherin [20], N-cadherin [20], CD133 [21], MMP-9 [22] and GAPDH [22] have been previously described. All miRNA and mRNA quantification data were normalized to GAPDH. Results were given as the fold change relative to controls.

\section{Cell proliferation assay and apoptosis assay}

To evaluate cell proliferation, $5 \times 10^{3}$ cells were plated in 96-well plates for $24 \mathrm{~h}$, and then transfected with miRNA mimic/inhibitor with or without Twist siRNA or Twist cDNA vector. At $72 \mathrm{~h}$, cell proliferation was determined by the Cell Counting Kit- 8 (Dojindo, Japan). The absorbance was determined at $450 \mathrm{~nm}$ using a microplate reader, and data were expressed as the percentage of absorbance relative to controls. For cell apoptosis assay, cells were transfected for $72 \mathrm{~h}$ and apoptotic cells were identified using the DeadEnd Colorimetric TUNEL System kit (Promega, WI, USA), following the manufacturer's instructions. Cell apoptosis was quantified as the numbers of apoptotic cells found in 15 random fields. The ratio of dead/total cell number was calculated.

\section{Cell migration and invasion assay}

Cells were transfected for $24 \mathrm{~h}$ and then seeded into upper chamber of Boyden chambers coated with or without Matrigel as described previously [23-25]. After incubation for $24 \mathrm{~h}$, migration and invasion were stained and counted under a light microscope. Relative migration and invasion activities were expressed as the fold change over respective controls.

\section{Western blot}

Cells were harvested $48 \mathrm{~h}$ after transfections. Whole-cell protein extracts were prepared using the M-Per Mammalian Protein Extraction Reagent (Pierce Biotechnology, MA, USA) according to the manufacturer's instructions. Total proteins $(40 \mu \mathrm{g})$ were loaded onto $10 \%$ SDS-PAGE for immunoblots with antibodies to Twist (Abcam, ab50887) and GAPDH (Santa Cruz, sc-47724). Primary antibodies were used at a dilution of 1:1000.

\section{Luciferase activity assay}

The Twist 3'-UTR luciferase vectors was obtained from OriGene (Rockville, MD, USA). A quick-change site- 
directed mutagenesis kit (Stratagene, CA, USA) was used to mutate the miR-548c-binding site. Luciferase activity was measured at $24 \mathrm{~h}$ after transfection using the dual-luciferase reporter assay system (Promega, WI, USA) as previously reported [15].

\section{Benign and tumor tissues}

50 pairs of primary EC and adjacent non-tumor endometrial tissues, 60 epithelial OC tissues and 20 normal epithelial ovarian tissues were collected in the China-Japan Union Hospital of Jilin University, China. This study was approved by the Ethics Committee of China-Japan Union Hospital of Jilin University and conducted according to the principles of the Declaration of Helsinki. The patients signed a written inform consent for the procedures in the study. Samples were snap-frozen immediately at $-80{ }^{\circ} \mathrm{C}$, and total RNA was isolated using TRIzol reagents.

\section{Statistical analysis}

Results are presented as mean \pm s.e.m. from at least three independent experiments performed in triplicate. 2-tailed Student's $t$-test was used for statistical analysis. $P$-values $<0.05$ were defined as significant.

\section{Results}

MiR-548c is downregulated in endometrial and ovarian cancer tissues and decreased miR-548c levels associates with worse prognosis in patients with endometrial cancer To examine the levels of miR-548c in EC tissues, we measured the endogenous miR-548c expression by realtime quantitative RT-PCR (qPCR) in $50 \mathrm{EC}$ samples and their adjacent normal tissues. Intriguingly, miR-548c was significantly decreased in ECs compared with adjacent normal tissues (Fig. 1a). We also analyzed miR-548c expression by qPCR in clinical samples of $60 \mathrm{OC}$ tissues (Table 1) compared to 20 normal ovarian tissues. a
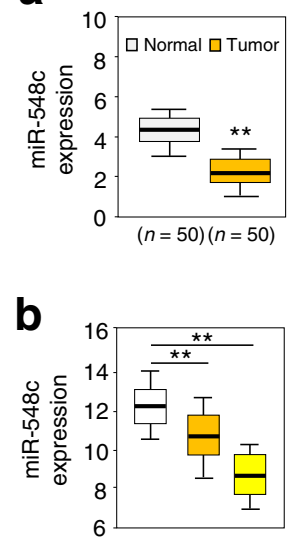

d

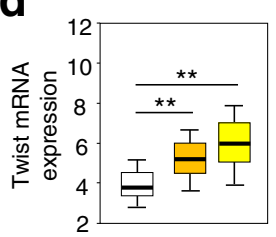

g

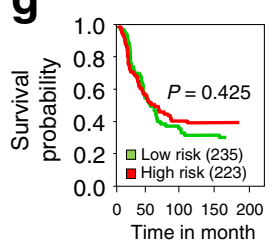

C
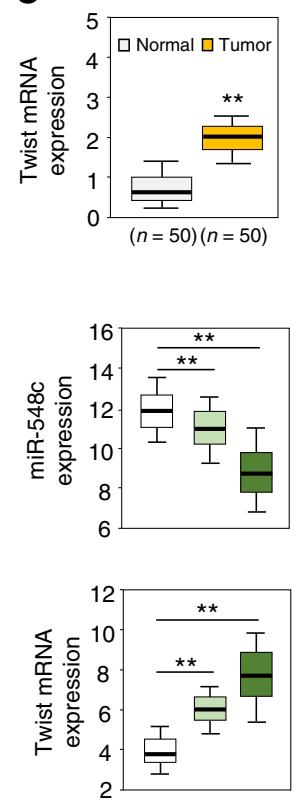

h

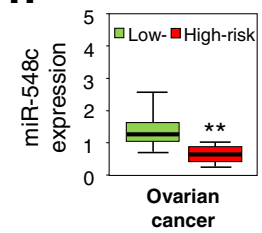

e
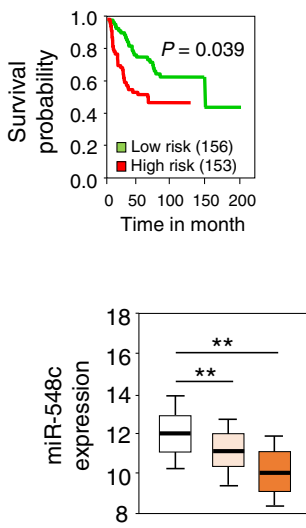

Ovarian cancer

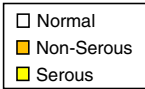

$\square$ Non-Serous
$\square$ Serous

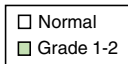

$\square$ Grade 3

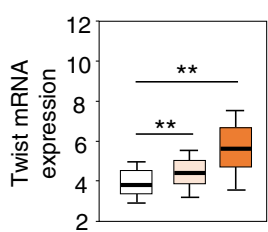

$\square$ Normal

$\square$ Stage III-IV

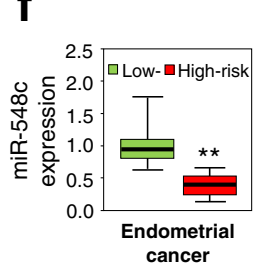

प Stage III-IV

Fig. 1 MiR-548c is downregulated in endometrial and ovarian cancer tissues and decreased miR-548c levels associates with worse prognosis in patients with endometrial cancer. Box plots showing decreased expression of miR-548c (a, b) and increased Twist (c, d) mRNA expression in 50 ECs and matched adjacent normal tissues (top), and in 60 OCs compared to 20 normal ovarian tissues (bottom), assessed by the quantitative realtime RT-PCR (qPCR) assays. Of note, downregulation of miR-548c (b) and upregulation of Twist (d) was detected in serous, advanced stage and grade 3 OC samples. Kaplan-Meier survival curves of EC (e) and OC (g) patients in The Cancer Genome Atlas (TCGA) data set were created using the SurvMicro database. In ECs, high-risk patients exhibited significantly lower levels of miR-548c compared to the low-risk patients (f). $\mathbf{h}$ MiR-548c expression between high-risk and low-risk OCs (TCGA). ${ }^{* *} P<0.01$ 
Decreased miR-548c expression was detected in OC tissues relative to normal ovarian tissues, and the expressions of miR-548c were significantly lower in serous, advanced stage and grade 3 tumor samples compared with non-serous, early stage and grade $1 / 2$ tumor samples (Fig. 1b), indicating that the deregulation of miR548 c may play a critical role in EC or OC development.

To investigate whether the downregulation of miR$548 \mathrm{c}$ is related with prognosis of EC or OC patients, we used the SurvMicro web tool [26] to investigate the relationship between miR-548c levels and prognosis of cancer patients. The analysis of published data on EC and OC from The Cancer Genome Atlas (TCGA) revealed that the low expression levels of miR-548c were associated with very poor overall survival in EC patients compared with those patients whose ECs express a high level of miR-548c (Fig. 1e and f). Although our meta-analysis of OCs using TCGA data did not show a clear separation between high-risk OCs and low-risk OCs based on miR-548c expression $(P=0.425)$, OC patients whose tumors display low miR-548c expression tend to have shorter survival (Fig. 1g and h). Thus, these data uncover an association between the loss of miR-548c expression and poor survival of EC patients, and indicate that miR$548 \mathrm{c}$ repression might be important for EC/OC growth and progression.

\section{MiR-548c inhibits proliferation, migration and invasion of EC and OC cells}

To select appropriate model for functional studies, we first evaluated miR-548c expression in two EC cell lines (RL95-2 and HEC-1) and OC cell line SKOV-3 using qPCRs. We found that invasive HEC-1 and SKOV-3 cells express much lower levels of miR-548c, compared with less invasive RL95-2 and OVCAR3 cells (Fig. 2a). The downregulation of miR-548c in cancer tissues and invasive cancer cells implicated that it might have the

Table 1 Clinical characteristics of 60 ovarian cancer patients

\begin{tabular}{ll}
\hline Features & Cases (\%) \\
\hline Age (years) & \\
$\leq 55$ & $32(53.3 \%)$ \\
$>55$ & $28(46.7 \%)$ \\
Clinical stage & \\
I-II & $19(31.7 \%)$ \\
III-IV & $41(68.3 \%)$ \\
Pathological grade & \\
$1-2$ & $24(40.0 \%)$ \\
3 & $36(60.0 \%)$ \\
Histotype & \\
Serous & $40(66.7 \%)$ \\
Non-serous & $20(33.3 \%)$ \\
\hline
\end{tabular}

tumor suppressive roles in the tumorigenesis and metastasis of EC and OC. To test this, we transiently transfected anti-miRNA inhibitor against miR-548c (anti-548c) or control inhibitor (anti-ctr) into RL95-2 or OVCAR3 cells, and then validated the depletion of miR-548c expression by qPCR assays (Fig. 2b). Furthermore, the ectopic overexpression of miR-548c in HEC-1 and SKOV-3 cells was achieved by transient transfection of pre-miR-548c mimic (pre-548c) (Fig. 2c). Then, cell proliferation was measured by the Cell Counting Kit- 8 assay. The downregulation of miR-548c in RL95-2 and OVCAR3 cells resulted in greater proliferation rate, whereas forced miR-548c expression significantly reduced proliferation of HEC-1 and SKOV-3 cells compared with the control mimic (pre-ctr) (Fig. 2d). To determine the effect of miR-548c on cell apoptosis, we examined apoptosis-associated DNA fragmentation by using a colorimetric TUNEL staining assay. MiR-548c knockdown decreased RL95-2 or OVCAR3 cell death, however miR-548c overexpression caused apoptotic cell death of HEC-1 and SKOV-3 cells (Fig. 2e). These results suggest that miR-548c inhibits EC and OC cell proliferation and triggers cell apoptosis.

To verify whether miR-548c affect the progression and metastasis of EC and OC, we evaluated the effect of either knockdown or overexpression of miR-548c on the migratory and invasive abilities of these cells. Transwell migration and invasion assays demonstrated that downregulation of miR-548c expression significantly promoted migration and invasion of RL95-2 and OVCAR3 cells (Fig. $2 \mathrm{f}$ and g). In contrast, transient overexpression of miR-548c in HEC-1 and SKOV-3 cells significantly impaired in vitro cell migration and invasion (Fig. 2f, g and $\mathrm{h}$ ). Together, these data support that miR-548c acts as a tumor suppressors to inhibit tumor growth, migration and invasion in both $\mathrm{EC}$ and $\mathrm{OC}$ cells.

\section{Twist is a direct target of MiR-548c in EC and OC cells}

To explore gene targets and molecular pathways that could be mediated by miR-548c, we performed computational target prediction using TargetScan and conducted in silico enrichment analysis of miR-548c-target genes in KEGG pathways using miRSystem [27]. Of note, miR-548c-regulated genes can potentially modulate numerous well-known pathways associated with tumor growth and metastasis, including pathways in cancer, mTOR pathway, adherens junction, cell cycle, acute myeloid leukemia, pancreatic cancer, notch signaling pathway and jak-stat signaling pathway (Fig. 3a), indicating the possibility that miR-548c has broad influence over diverse oncogenic pathways. These findings are consistent with our results (Fig. 2), supporting the roles of miR-548c to suppress multiple malignant phenotypes of EC and OC cells including proliferation, migration and invasion. 


\section{a}

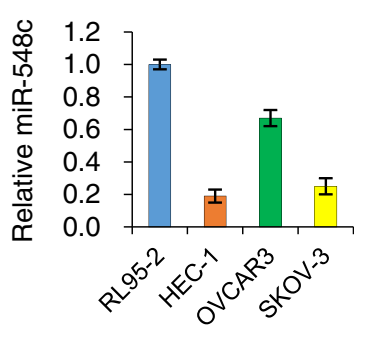

d

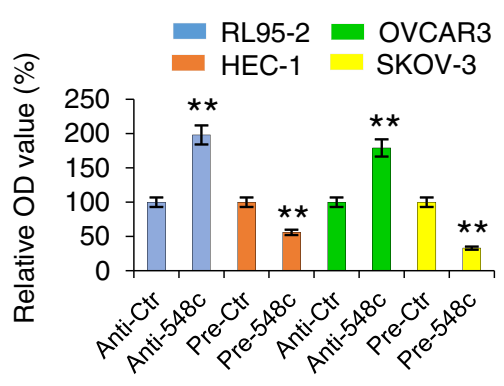

f

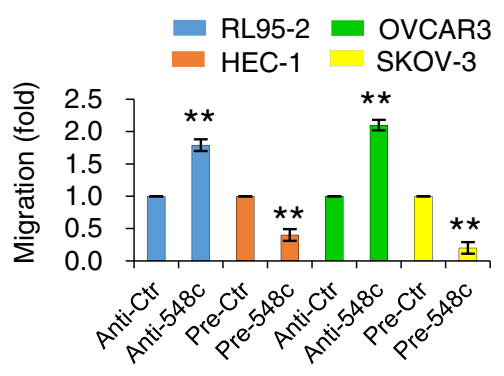

h

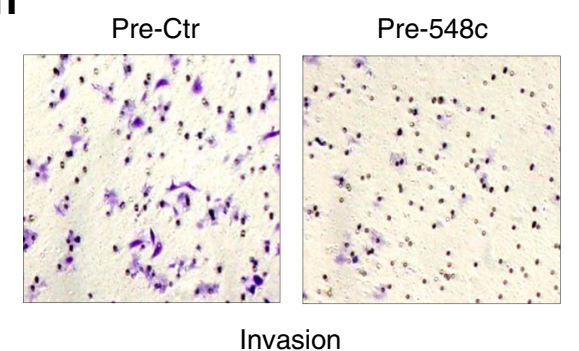

b
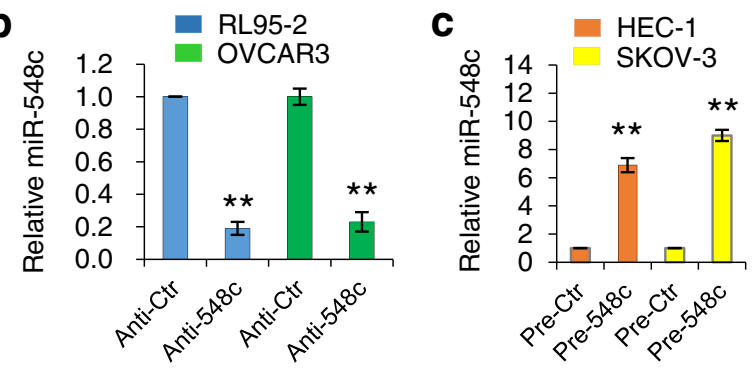

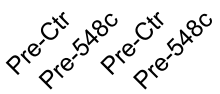
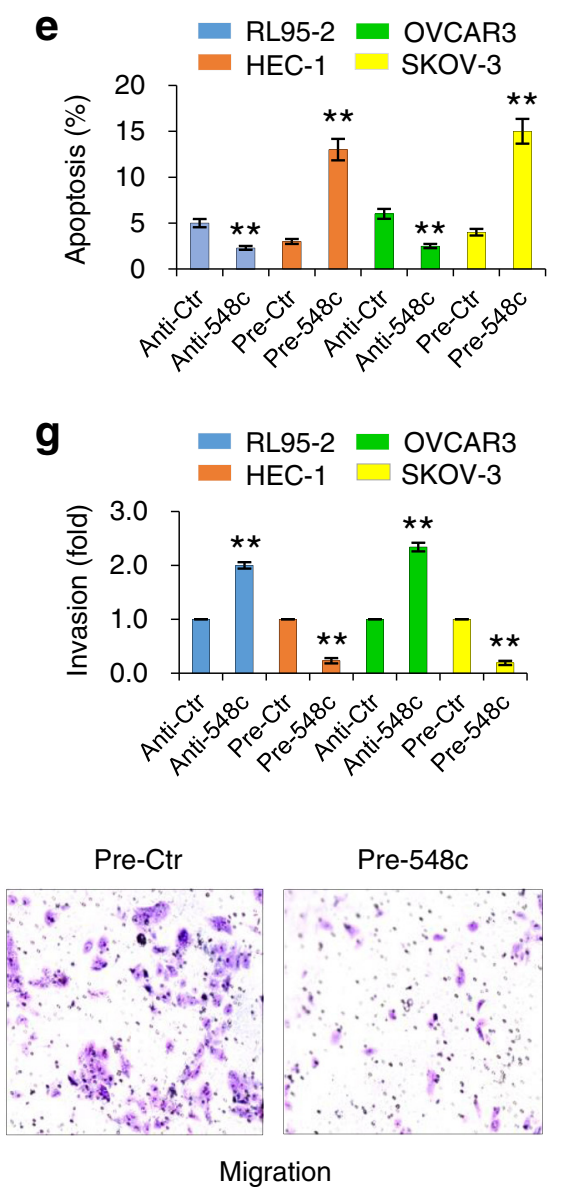

Fig. 2 MiR-548C inhibits proliferation, migration and invasion of EC and OC cells. a Relative expression of miR-548c in two EC cell lines (RL95-2 and HEC-1) and OC cell lines (OVCAR3 and SKOV-3 was determined by qPCRs. Data is presented as the fold-change in expression compared with RL95-2. b qPCR analysis of miR-548c expression in RL95-2 and OVCAR3 cells transfected with anti-miR-548c inhibitor (anti-548c) or control inhibitor (anti-ctr). c miR-548c levels were measured by qPCRs in HEC-1 or SKOV-3 cells after overexpression of pre-miR-548c mimic (pre-548c) or control mimic (pre-ctr). Cell proliferation assay (d), cell apoptosis assay (e), transwell migration (f) and matrigel invasion (g) assays were performed in indicated cells after knockdown or overexpression of miR-548c, respectively. $\mathbf{h}$ Representative images from cell migration and invasion assays performed in SKOV-3 cells. ${ }^{* *} P<0.01$

Because Twist was identified as a potential target of miR-548c (Fig. 3b), we speculated that Twist serves as a direct target of miR-548c. Our western blot analysis suggested that the introduction of anti-548c led to an increase in Twist protein level in RL95-2 cells, while pre-548c resulted in the opposite effects in HEC-1 and SKOV-3 cells (Fig. 3c). To test whether miR-548c repression is mediated through the predicted binding site, the reporter plasmid containing putative miR-548c responsive site was transfected with anti-548c or anti-ctr into RL95-2 cells. The downregulation of miR-548c enhanced Twist 3'-UTR luciferase reporter activity compared with the negative control (Fig. 3d, left), suggesting that miR-548c suppresses translation of Twist. To verify that miR-548c directly represses Twist on its 3'-UTR, we cotransfected pre-548c or pre-ctr with the Twist 3'-UTR 
a

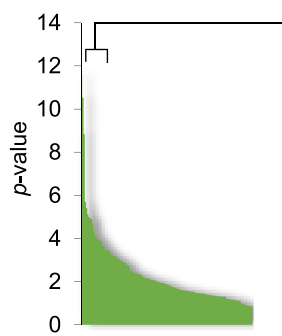

\begin{tabular}{llll}
\hline No. Annotated KEGG Pathway & No. & Annotated KEGG Pathway \\
\hline 1 & ENDOCYTOSIS & 11 & INSULIN_SIGNALING_PATHWAY \\
2 & PATHWAYS_IN_CANCER & 12 & SPLICEOSOME \\
3 & UBIQUITIN_MEDIATED_PROTEOLYSIS & 13 & ADIPOCYTOKINE_SIGNALING_PATHWAY \\
4 & ADHERENS_JUNCTION & 14 & BACTERIAL_INVASION_OF_EPITHELIAL_CELLS \\
5 & MTOR_SIGNALING_PATHWAY & 15 & PANCREATIC_CANCER \\
6 & ALZHEIMER'S_DISEASE & 16 & TIGHT_JUNCTION \\
7 & CELL_CYCLE & 17 & VASOPRESSIN-REGULATED_WATER_REABSORPTION \\
8 & ACUTE_MYELOID_LEUKEMIA & 18 & NOTCH_SIGNALING_PATHWAY \\
9 & REGULATION_OF_AUTOPHAGY & 19 & JAK-STAT_SIGNALING_PATHWAY \\
10 & CHRONIC_MYELOID_LEUKEMIA & 20 & CYSTEINE_AND_METHIONINE_METABOLISM \\
\hline
\end{tabular}

b

\begin{tabular}{|c|c|c|c|c|}
\hline Gene & Conservation & 3'UTR position & Target Site & Target Prediction Program \\
\hline Twist1 & $\begin{array}{l}\mathrm{H} / \mathrm{M} \\
\mathrm{P} / \mathrm{C}\end{array}$ & $636-642$ & $\begin{array}{l}5^{\prime} \ldots \text { TCTAAATGCATTCATATTTTTAT ... (Wild-type UTR) } \\
3^{\prime} \ldots \text { CGUUUUCAUUAACUC UAAAAAC... (miR-548c-3p) } \\
5^{\prime} \ldots \text { TCTAAATGCATTCATGCCCCCAT ... (Mutant UTR) }\end{array}$ & TargetScan \\
\hline
\end{tabular}

C

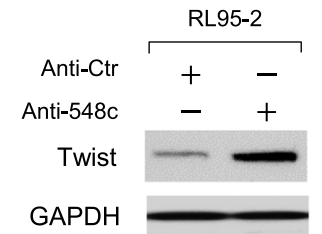

d

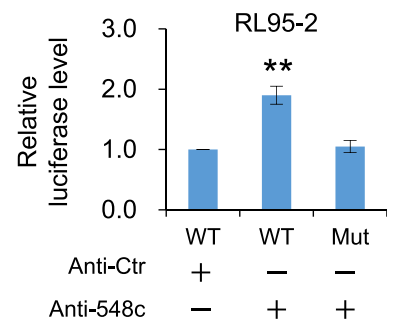

e

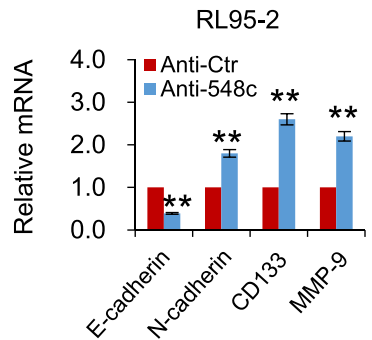

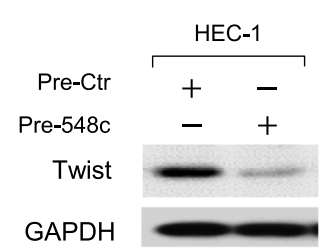
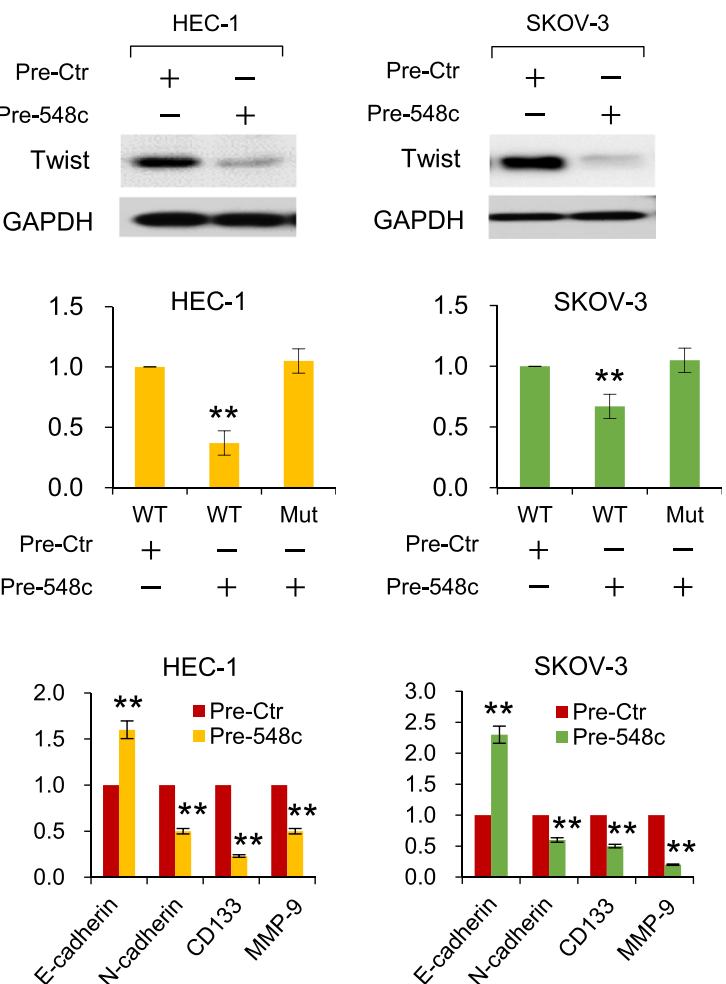

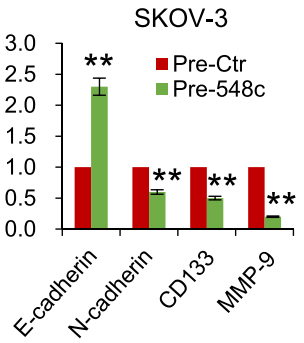

Fig. 3 Twist is a direct target of miR-548c in EC and OC cells. a In silico prediction and molecular pathway enrichment analysis indicate that miR548c-regulated genes are preferentially involved in pathway associated with tumor growth and metastasis. The top-20 ranking KEGG pathways were listed. $\mathbf{b}$ The putative conserved miR-548c-binding site in the Twist 3'-UTR. Mutation was generated in the binding site. $\mathbf{c}$ Western blotting analysis of Twist expression in RL95-2, HEC-1 or SKOV-3 cells after knockdown or overexpression of miR-548c. d Indicated cells were transfected with reporter constructs containing either wild-type (WT) Twist, or Twist 3'-UTR with mutation (Mut), along with miR-548c mimic, anti-miR-548c inhibitor or negative controls, respectively. Relative luciferase activity was assayed. e qPCR analysis of EMT, invasion and stemness-related genes in RL95-2 upon miR-548c knockdown, or in HEC-1 or SKOV-3 cells after overexpression of miR-548c. ${ }^{* *} P<0.01$

reporter into HEC-1 and SKOV-3 cells. The ectopic expression of pre-548c caused a repression on luciferase activity as compared with pre-ctr (Fig. 3d, middle and right). To further evaluate the interaction between miR548c and Twist 3'-UTR, we generated Twist 3'-UTR mutated for miR-548c binding site and performed the luciferase assays upon either miR-548c overexpression or knockdown. Mutant 3'-UTR reporter activity was resistant to the addition of anti-548c or pre-548c (Fig. 3d), confirming that Twist is a direct target of miR$548 \mathrm{c}$, and binding of miR-548c to the 3'-UTR of Twist mRNA is sufficient to reduce Twist protein levels.

Twist binds the E-cadherin promoter and represses E-cadherin transcription, whereas directly activating $\mathrm{N}$-cadherin expression $[28,29]$. In addition, the EMT induction has been linked to the emergence of a 
cancer stem cell (CSC)-like phenotype [30]. Consistent with this notion, sorted $\mathrm{CD}_{133^{+}}$EC cells have been shown to demonstrate greater tumor initiating capacity than $\mathrm{CD} 133^{-}$cells [31], and CSC-like cells obtained from SKOV-3 cells express higher levels of CD133 [32]. Matrix metalloproteinase 9 (MMP-9) is a downstream target of Twist in tumor cells [33]. We test whether miR-548c regulates these genes in cancer cells used in this study. By using qPCR, we found a significant decrease in the expression of E-cadherin and an increased expression in N-cadherin, CD133 and MMP-9 levels in anti-548c-transfected RL95-2 cells (Fig. 3e). In contrast, miR-548c overexpression in HEC-1 and SKOV-3 cells significantly induced $E$ cadherin levels, but inhibited the expression of $\mathrm{N}$-cadherin, CD133 and MMP-9 (Fig. 3e). Furthermore, we used qPCR to examine the mRNA expression of Twist in EC and OC tissues. As expected, Twist levels was markedly elevated in EC samples when compared to adjacent normal tissues (Fig. 1c). We also observed significantly increased Twist expression in OCs relative to normal ovarian tissues, and found higher levels of Twist in serous, advanced, high-grade ovarian cancers (Fig. 1d). Altogether, these data are consistent with our hypothesis that increased Twist expression in $\mathrm{EC}$ and $\mathrm{OC}$ is a functional consequence of miR$548 \mathrm{c}$ repression (at least in part).

\section{Twist mediates miR-548c-reduced cancer cell migration and invasion}

To further study whether miR-548c can attenuate oncogenic phenotypes in EC and OC cells through downregulation of Twist, we performed cell proliferation, migration and invasion assays using EC or OC cells transduced with anti-548c or pre-548c, along with either Twist siRNA or Twist cDNA expression plasmid without 3'-UTR sequence, which renders the escape from miR-548c regulation. Anti-548c-induced RL95-2 cell proliferation, migration and invasion were significantly abrogated by Twist siRNA (Fig. 4a, c and e). On the other hand, overexpression of Twist cDNA largely rescued pre-548c-suppressed proliferation, migration and invasion in HEC-1 cells (Fig. 4b, d and f, left). Although expression of Twist cDNA restored SKOV-3 cell migration and invasion that were reduced by pre-548c (Fig. $4 \mathrm{~d}$ and $\mathrm{f}$, right), cell proliferative phenotype was not significantly restored by Twist overexpression in SKOV-3 cells (Fig. 4b, right), consistent with a previous report showing that Twist confers migratory and invasive potential, but does not affect proliferation of OC cells [8].

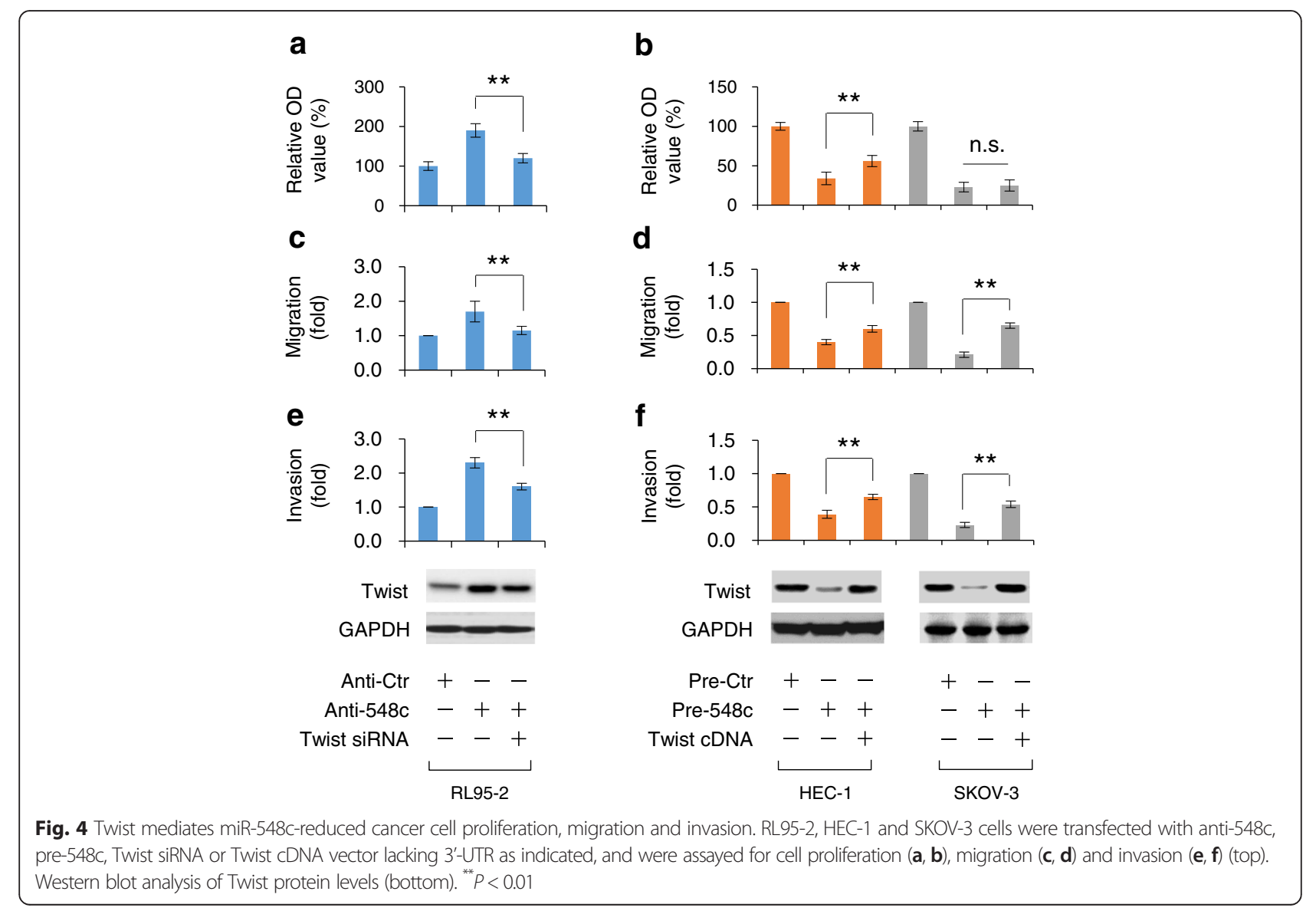


Collectively, these data support the mechanistic roles for miR-548c in impairing migration and invasion of EC and $\mathrm{OC}$ cells by targeting Twist.

\section{Discussion}

Targeting of metastasis-related miRNAs in human tumors may offer potential therapeutic strategies for these diseases [34, 35]. Recent findings have identified miR$548 \mathrm{c}$ family members as universe miRNA, and illustrated the possibility that these miRNAs exert broad effects on many features of tumor cells through a broad range of targets and pathways [17]. Although one such miRNA, miR-548c, has been shown to inhibit cancer cell growth [19], its cellular functions, underlying mechanisms and clinical significance have not been comprehensively studied in gynecological cancers. Here, we found that miR-548c was under-expressed in both EC and OC samples, compared to their non-tumor counterparts. At the molecular level, miR-548c represses migration and invasion of EC and OC cells partially by targeting Twist. We demonstrate that miR-548c expression was inversely correlated with Twist level in EC and OC tissues, and reduced miR-548c expression was associated with poor prognosis in EC patients. These findings reveal for the first time that in EC and OC cells, miR-548c is a tumorsuppressive miRNA that inhibits multiple malignant features of the metastatic cascade.

Twist has been known as a novel oncogene overexpressed in diverse tumors [36], and activation of Twist inhibits apoptosis and promotes the induction of EMT, cancer stemness, proliferation, angiogenesis and vasculogenic mimicry in human tumor cells, all of which contributes to metastasis $[37,38]$. Several upstream regulators and downstream effector of Twist have been reported [37], however our knowledge of the detailed molecular mechanisms involved in Twist overexpression, especially the epigenetic regulation of Twist expression, is still incomplete. Here, we reported a previously unidentified mechanism whereby loss of miR-548c expression is partially responsible for increased Twist levels in EC and OC, two of the most common gynecologic cancers.

Twist has been shown to upregulate the expression of some miRNAs, such as miR-10b [39] and miR-424 [40]. Twist promotes the expression of miR-10b by directly binding to the promoter of miR-10b, which initiates tumor invasion and metastasis in breast cancer [39]. Furthermore, miR-424, another miRNA that can be induced by Twist, drives EMT-like phenotypes in breast cancers [40]. The impact of Twist on miR-548c expression in EC and OC cells warrants further investigation.

MiR-548c was reported to be an independent prognostic factor for breast cancer. Patients with a good prognosis presented higher intratumoral expression of miR-548c [41]. Consistently, our meta-analysis suggested that high expression of miR-548c in EC and OC appears to correlate with better survival in patients with $\mathrm{EC}$ and $\mathrm{OC}$, implying that miR-548c may be a new promising prognostic factor in these cancers. Since miR-548c directly regulates Twist in EC and OC cells, this miRNA might also represent an attractive target for the treatment of Twist-overexpressing tumors.

\section{Conclusions}

Our findings provide a novel mechanism by which the repression of miR-548c increases Twist levels to stimulate cancer cell migration and invasion of both $\mathrm{EC}$ and OC cells. Our results indicate that miR-548c is a potential therapeutic target in EC and OC.

\section{Competing interests}

The authors declare no competing financial interests.

\section{Authors' contributions}

MC designed experiments. XS, AZ and LT conducted all experiments. MC and $\mathrm{XS}$ wrote the manuscript. AZ, LT, KW, KL, XW, ZS and $\mathrm{HZ}$ contributed to data analysis and discussed the results. All authors read and approved the final manuscript.

\section{Acknowledgements \\ We are grateful to the staff and patients at the Department of Obstetrics and Gynecology, China-Japan Union Hospital of Jilin University for providing the samples.}

\section{Author details}

${ }^{1}$ Department of Obstetrics and Gynecology, China-Japan Union Hospital of Jilin University, Changchun 130021, China. 'Department of Obstetrics and Gynecology, the Second Hospital of Jilin University, Changchun 130041, China.

Received: 27 October 2015 Accepted: 8 January 2016

Published online: 13 January 2016

References

1. Dong P, Kaneuchi M, Konno Y, Watari H, Sudo S, Sakuragi N. Emerging therapeutic biomarkers in endometrial cancer. Biomed Res Int. 2013;2013: 130362

2. Kim A, Ueda Y, Naka T, Enomoto T. Therapeutic strategies in epithelial ovarian cancer. J Exp Clin Cancer Res. 2012;31:14.

3. Bast Jr RC, Hennessy B, Mills GB. The biology of ovarian cancer: new opportunities for translation. Nat Rev Cancer. 2009;9:415-28.

4. Mehlen P, Puisieux A. Metastasis: a question of life or death. Nat Rev Cancer. 2006;6:449-58.

5. Vergara D, Merlot B, Lucot JP, Collinet P, Vinatier D, Fournier I, et al. Epithelial-mesenchymal transition in ovarian cancer. Cancer Lett. 2010;291: 59-66.

6. Kaufhold S, Bonavida B. Central role of Snail1 in the regulation of EMT and resistance in cancer: a target for therapeutic intervention. J Exp Clin Cancer Res. 2014;33:62

7. Wang WS, Yu SL, Yang XS, Chang SD, Hou JQ. Expression and significance of twist and E-cadherin in ovarian cancer tissues. Asian Pac J Cancer Prev. 2013;14:669-72.

8. Wang WS, Yang XS, Xia M, Jiang HY, Hou JQ. Silencing of twist expression by RNA interference suppresses epithelial-mesenchymal transition, invasion, and metastasis of ovarian cancer. Asian Pac J Cancer Prev. 2012;13:4435-9.

9. Kyo S, Sakaguchi J, Ohno S, Mizumoto Y, Maida Y, Hashimoto M, et al. High Twist expression is involved in infiltrative endometrial cancer and affects patient survival. Hum Pathol. 2006:37:431-8.

10. Yoshida J, Horiuchi A, Kikuchi N, Hayashi A, Osada R, Ohira S, et al. Changes in the expression of E-cadherin repressors, Snail, Slug, SIP1, and Twist, in the development and progression of ovarian carcinoma: the important role of 
Snail in ovarian tumorigenesis and progression. Med Mol Morphol. 2009;42: 82-91.

11. Hosono S, Kajiyama H, Terauchi M, Shibata K, Ino K, Nawa A, et al. Expression of Twist increases the risk for recurrence and for poor survival in epithelial ovarian carcinoma patients. Br J Cancer. 2007;96:314-20.

12. Pencheva N, Tavazoie SF. Control of metastatic progression by microRNA regulatory networks. Nat Cell Biol. 2013;15:546-54.

13. Liu X, Chen X, Yu X, Tao Y, Bode AM, Dong Z, et al. Regulation of microRNAs by epigenetics and their interplay involved in cancer. J Exp Clin Cancer Res. 2013;32:96

14. Katz B, Tropé CG, Reich R, Davidson B. MicroRNAs in ovarian cancer. Hum Pathol. 2015;46:1245-56.

15. Dong P, Kaneuchi M, Watari H, Sudo S, Sakuragi N. MicroRNA-106b modulates epithelial-mesenchymal transition by targeting TWIST1 in invasive endometrial cancer cell lines. Mol Carcinog. 2014;53:349-59.

16. Bing L, Hong C, Li-Xin S, Wei G. MicroRNA-543 suppresses endometrial cancer oncogenicity via targeting FAK and TWIST1 expression. Arch Gynecol Obstet. 2014;290:533-41.

17. Shirdel EA, Xie W, Mak TW, Jurisica I. NAViGaTing the micronome-using multiple microRNA prediction databases to identify signalling pathwayassociated microRNAs. PLoS ONE. 2011;6:e17429.

18. Piriyapongsa J, Jordan IK. A family of human microRNA genes from miniature inverted-repeat transposable elements. PLoS ONE. 2007;2, e203.

19. Hu B, Ying X, Wang J, Piriyapongsa J, Jordan IK, Sheng J, et al. Identification of a tumor-suppressive human-specific microRNA within the FHIT tumorsuppressor gene. Cancer Res. 2014;74:2283-94.

20. Odero-Marah VA, Wang R, Chu G, Zayzafoon M, Xu J, Shi C, et al. Receptor activator of NF-kappaB Ligand (RANKL) expression is associated with epithelial to mesenchymal transition in human prostate cancer cells. Cell Res. 2008;18:858-70.

21. Chiou SH, Wang ML, Chou YT, Chen CJ, Hong CF, Hsieh WJ, et al. Coexpression of Oct4 and Nanog enhances malignancy in lung adenocarcinoma by inducing cancer stem cell-like properties and epithelialmesenchymal transdifferentiation. Cancer Res. 2010;70:10433-44.

22. Figueira RC, Gomes LR, Neto JS, Silva FC, Silva ID, Sogayar MC. Correlation between MMPs and their inhibitors in breast cancer tumor tissue specimens and in cell lines with different metastatic potential. BMC Cancer. 2009;9:20.

23. Dong P, Kaneuchi M, Watari H, Hamada J, Sudo S, Ju J, et al. MicroRNA-194 inhibits epithelial to mesenchymal transition of endometrial cancer cells by targeting oncogene BMI-1. Mol Cancer. 2011;10:99.

24. Dong P, Karaayvaz M, Jia N, Kaneuchi M, Hamada J, Watari H, et al. Mutant p53 gain-of-function induces epithelial-mesenchymal transition through modulation of the miR-130b-ZEB1 axis. Oncogene. 2013;32:3286-95.

25. Dong PX, Jia N, Xu ZJ, Liu YT, Li DJ, Feng YJ. Silencing of IQGAP1 by shRNA inhibits the invasion of ovarian carcinoma HO-8910PM cells in vitro. J Exp Clin Cancer Res. 2008;27:7.

26. Aguirre-Gamboa R, Trevino V. SurvMicro: assessment of miRNA based prognostic signatures for cancer clinical outcomes by multivariate survival analysis. Bioinformatics. 2014;30:1630-2.

27. Lu TP, Lee CY, Tsai MH, Chiu YC, Hsiao CK, Lai LC, et al. miRSystem: an integrated system for characterizing enriched functions and pathways of microRNA targets. PLoS ONE. 2012;7:e42390.

28. Vesuna $\mathrm{F}$, van Diest $\mathrm{P}$, Chen $\mathrm{JH}$, Raman $\mathrm{V}$. Twist is a transcriptional repressor of E-cadherin gene expression in breast cancer. Biochem Biophys Res Commun. 2008:367:235-41.

29. Alexander NR, Tran NL, Rekapally H, Summers CE, Glackin C, Heimark RLAlexander NR, et al. N-cadherin Gene Expression in Prostate Carcinoma Is Modulated by Integrin-Dependent Nuclear Translocation of Twist1. Cancer Res. 2006;66:3365-9.

30. Singh A, Settleman J. EMT, cancer stem cells and drug resistance: an emerging axis of evil in the war on cancer. Oncogene. 2010;29:4741-51.

31. Nakamura M, Kyo S, Zhang B, Zhang X, Mizumoto Y, Takakura M, et al. Prognostic impact of CD133 expression as a tumor-initiating cell marker in endometrial cancer. Hum Pathol. 2010:41:1516-29.

32. Ma L, Lai D, Liu T, Cheng W, Guo L. Cancer stem-like cells can be isolated with drug selection in human ovarian cancer cell line SKOV3. Acta Biochim Biophys Sin (Shanghai). 2010;42:593-602.

33. Zhao XL, Sun T, Che N, Sun D, Zhao N, Dong XY, et al. Promotion of hepatocellular carcinoma metastasis through matrix metalloproteinase activation by epithelial-mesenchymal transition regulator Twist1. J Cell Mol Med. 2011;15:691-700
34. Brunetti O, Russo A, Scarpa A, Santini D, Reni M, Bittoni A, et al. MicroRNA in pancreatic adenocarcinoma: predictive/prognostic biomarkers or therapeutic targets? Oncotarget. 2015;6:23323-41.

35. Luo G, Chao YL, Tang B, Li BS, Xiao YF, Xie R, et al. miR-149 represses metastasis of hepatocellular carcinoma by targeting actin-regulatory proteins PPM1F. Oncotarget. 2015;6:37808-23.

36. Kang Y, Massagué J. Epithelial-mesenchymal transitions: twist in development and metastasis. Cell. 2004;118:277-9.

37. Khan MA, Chen HC, Zhang D, Fu J. Twist: a molecular target in cancer therapeutics. Tumour Biol. 2013;34:2497-506.

38. Jung HY, Yang J. Unraveling the TWIST between EMT and Cancer Stemness. Cell Stem Cell. 2015;16:1-2.

39. Ma L, Teruya-Feldstein J, Weinberg RA. Tumour invasion and metastasis initiated by microRNA-10b in breast cancer. Nature. 2007;449(7163):682-8.

40. Drasin DJ, Guarnieri AL, Neelakantan D, Kim J, Cabrera JH, Wang CA, et al. TWIST1-Induced miR-424 Reversibly Drives Mesenchymal Programming while Inhibiting Tumor Initiation. Cancer Res. 2015;75(9):1908-21.

41. Boukerroucha M, Josse C, ElGuendi S, Boujemla B, Frères $P$, Marée R, et al. Evaluation of BRCA1-related molecular features and microRNAs as prognostic factors for triple negative breast cancers. BMC Cancer. 2015;15:755.

\section{Submit your next manuscript to BioMed Central and we will help you at every step:}

- We accept pre-submission inquiries

- Our selector tool helps you to find the most relevant journal

- We provide round the clock customer support

- Convenient online submission

- Thorough peer review

- Inclusion in PubMed and all major indexing services

- Maximum visibility for your research

Submit your manuscript at www.biomedcentral.com/submit
() Biomed Central 